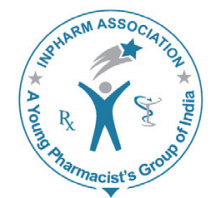

\title{
Design and Development of a novel transmucosal patch Embedded with Diclofenac Diethylamine loaded solid lipid nanoparticles
}

\author{
Nidhi Malviyaa, Kusumvalli Somisetty ${ }^{\mathrm{b}}$, Kusumdevi Vemula ${ }^{\mathrm{b}^{*}}$ \\ ${ }^{a}$ Department of Pharmaceutics, Al-Ameen College of Pharmacy, Hosur Road, Bangalore \\ ${ }^{b}$ Department of Endodontics and Conservative Dentistry, Sri Rajiv Gandhi College of Dental Sciences, \\ Cholanagar, Bangalore, Karnataka, India
}

\begin{abstract}
Objective: Diclofenac diethylamine (DDEA) loaded solid lipid nanoparticles (SLN) were developed and incorporated in to transmucosal patch (TP) for application in dental surgeries. Methods: DDEA-SLNs were prepared by solvent emulsion evaporation method with compritol 888 ATO as lipid and soya lecithin, Pluronic F68 as surfactants and optimized by a 3-factor 3-level central composite design for its impact on particle size (PS) and entrapment efficiency (EE). SLN was incorporated into a bilayer TP prepared with hydroxypropyl cellulose - LF and polycarbophil along with Labrafac as plasticizer. TP was characterized for tensile and mucoadhesive strength, FTIR, DSC, XRD, SEM, in vitro and ex vivo release. Results: PS, EE, in vitro and ex vivo release of the optimized SLN batch D6 were found to be $178.88 \pm 1.3 \mathrm{~nm}, 54.14 \pm 1.6 \%, 98.26 \pm 3.4 \%$ and $96.28 \pm 3.5 \%$ at $24 \mathrm{~h}$ respectively. TP showed $99.22 \pm 0.7 \%$ of in vitro release and $99.53 \pm 0.9 \%$ permeation through porcine mucosa at $24 \mathrm{~h}$ with satisfactory tensile strength and mucoadhesive properties. Conclusion: The designed once a day TP loaded with DDEA-SLN applied at the gingival site, immediately after dental surgery has the potential to produce therapeutic relief locally which is prolonged for $24 \mathrm{~h}$, with the added advantage of overcoming first pass metabolism and gastric irritation, in addition to decreasing the frequency of administration of Diclofenac.
\end{abstract}

Key words: Ex-vivo permeation, Statistical optimization, Diclofenac SLN, Transmucosal patch.

\begin{tabular}{|l|l|}
\hline \multicolumn{2}{|c|}{ Access this article online } \\
\hline \multirow{2}{*}{ Journal Sponsor } & \\
\hline & $\begin{array}{l}\text { Website: } \\
\text { www.jyoungpharm.org }\end{array}$ \\
\cline { 2 - 3 } & DOI: \\
& $10.5530 /$ jyp.2015.1.8 \\
& \\
\hline
\end{tabular}

\section{INTRODUCTION}

Dental procedures such as deep tooth scaling and, root canal treatment, require establishment of optimal serum levels of NSAID preoperatively and while tissue remains anesthetized for prevention and treatment of acute pain. ${ }^{1}$ Diclofenac is extensively prescribed. ${ }^{2}$ and is available as suppositories, suspensions, syrups, capsules, tablets and injectables. Suppositories are unacceptable to certain patients, difficult to administer by arthritis patients and have

\footnotetext{
*Address for correspondence:

Dr. Kusumdevi, Vemula, M-Pharm, Ph. D, Professor and Head, Vice Principal, Department of Pharmaceutics, Al-Ameen College of Pharmacy, Hosur Road, Bangalore-27,E-mail: kusumdevivemula@gmail.com
} 
unpredictable and variable absorption. In case of solutions and suspensions the problems are with dose precision, stability, microbial contamination, and bulky to handle. Capsules and tablets of Diclofenac undergo first pass metabolism, cause gastric irritation, delayed onset of action and the frequency of administration is more than once a day. Limitation of injectables is pain due to prick of needle. These dosage forms do not address local analgesic effect for prolonged period of $24 \mathrm{~h}$ in post operative dental pain

Solid lipid nanoparticle (SLN) are colloidal carriers (50$1000 \mathrm{~nm}$ ) which increase the bioavailability of drug, ${ }^{3}$ and remain in a solid state at room and body temperature. SLN haveadvantages of high drug loading for lipophilic drugs, controlled release, ease of preparation and scale up and high biocompatibility. currently marketed above described dosage forms we aimed to prepare a bilayer transmucosal patch (TP) incorporated with Diclofenac diethylamine (DDEA) SLN which will have the advantage of unidirectional release of the drug, local delivery for prolonged period, avoiding of first pass metabolism and gastric irritation. Once a day application would decrease the frequency of administration.

\section{MATERIALS AND METHODS}

DDEA from Neon Pharmaceutical Ltd, Compritol ATO 888 (Compritol) and Labrafac ${ }^{\text {TM }}$ PG (Propylene glycol dicaprylate/dicaprate) (LPG) from Gattefosse, Ethyl cellulose (EC) (viscosity 20 cps with 50\% ethoxy content) from Dow cellulosic, Noveon $\mathbb{R}$ AA-1 Polycarbophil(PC) from Lubrizol were generous gift samples. Pluronic F 68 (PF68) from Sigma Aldrich, Soya lecithin (SL), dichloromethane (DCM) and dialysis membrane (MWCO 12,000-14,000) from Himedia and Dibutyl phthalate (DBP), Ethyl acetate (EA), Acetone and Hydroxy propyl cellulose LF (HPC-LF) from Loba Chem were purchased. Milli Q water was used for preparation and all other chemicals were of analytical grade.

\section{Selection of lipid}

Thermal characteristic behavior of DDEA, Comp and mixture of DDEA $(10 \mathrm{mg})$ in comptol $(100 \mathrm{mg})$ were studied. The mixture was melted above the melting point of lipid $\left(72.48^{\circ} \mathrm{C}\right)$ subjecting to Differential Scanning colourimetry (DSC) studies. ${ }^{4}$

\section{Preparation of DDEA-SLN}

DDEA-SLN was prepared by solvent emulsion evaporation technique (SEET). ${ }^{5}$ Briefly, DDEA $(36.72 \% \mathrm{w} / \mathrm{w}$ of total solids), Compritol (0.5-1.5\% w/v) and SL (12.66\% w/w) were dissolved in 10-25 ml of DCM. This was added $(1 \mathrm{ml} / \mathrm{min})$ into $50 \mathrm{ml}$ of aqueous hot solution $\left(80^{\circ} \mathrm{C}\right)$ of PF68 (0.1-0.15\% w/v) with stirring (Ultra Turrax IKAT25 Germany) at 20000 RPM for 30 min. Resulting nanodispersion was cooled at room temperature. DDEASLN was separated by centrifugation at $17000 \mathrm{rpm}$ (Remi Centrifuge CPR-24, India) for $30 \mathrm{~min}$. at $4^{\circ} \mathrm{C}$. The supernatant containing un-entrapped drug was discarded. The residue was washed to remove traces of free drug and surfactant and redispersed in water. The dispersion was frozen at $-40^{\circ} \mathrm{C}$ and vacuum dried (Operon, Japan).

\section{Experimental Design}

The central composite design (CCD) was generated by Design Expert software (version 8.0.7 Stat-Ease Inc., Minnesota) to obtain least particles size (PS) and maximum entrapment efficiency (EE). The design recommended 20 experimental trials at two-levels including runs at 8 factorial, 6 axial and 6 replicate as the Center Point for the estimation. The critical independent variables, $\mathrm{A}(0.5-1.5 \%$ $\mathrm{w} / \mathrm{v}$ lipid), B (0.1-0.15\% w/v \% surfactant), and C (10-25 $\mathrm{ml}$ Volume of organic phase) were studied at: $-\alpha$, low $(-1)$, middle (0), high (1) and $+\alpha$ levels whereas the dependent variables were. PS $\left(\mathrm{Y}_{1}\right)$ and $\mathrm{EE}\left(\mathrm{Y}_{2}\right)$. The value $\alpha$ at \pm 1.682 was considered to fulfill ratability and orthogonally aspects in the design. Predicting the response by the second-order polynomial equation resulted in Equation 1:

$Y=\left(\alpha_{0}+\alpha_{1} A+\alpha_{2} B+\alpha_{3} C+\alpha_{11} A^{2}+\alpha_{22} B^{2} \alpha_{33} C^{2}+\alpha_{12}\right.$ $\left.A B+\alpha_{13} A C+\alpha_{23} B C\right)$

Where, $\mathrm{Y}$ is the predicted response, $\alpha_{0}$ is the intercept; $\alpha_{1}, \alpha_{2}$ and $\alpha_{3}$ are the linear coefficients; $\alpha_{11}, \alpha_{22}$, and $\alpha_{33}$ are the squared coefficients; $\alpha_{12}, \alpha_{13}$ and $\alpha_{23}$ are the interaction coefficients.

\section{Determination of PS and zeta potential (ZP) of DDEA-SLN}

An aliquot of lyophilized SLN was resuspended in Milli $\mathrm{Q}$ water and the mean PS, poly dispersity index (PDI) and ZP was determined by Zetasizer Nano ZS90 (Malvern Instruments, UK).

\section{Determination of EE of DDEA-SLN}

Accurately weighed amount of DDEA-SLN was dispersed in distilled water and centrifuged at $17000 \mathrm{rpm}$ for $30 \mathrm{~min}$. The sediment were dissolved in DCM a common solvent for drug and lipid, and analysed spectrophotometrically at $282 \mathrm{~nm}$ (Shimadzu, Japan) for entrapped DDEA ( $\left.\mathrm{E}_{\text {DDEA }}\right)$.

Journal of Young Pharmacists Vol 7 • Issue 1 • Jan-Mar 2015 
EE and Drug loading (DL) percentage was calculated ${ }^{7}$ according to equation no 2 and 3 respectively.

$$
\begin{aligned}
& \operatorname{DL}(\%)=\left\{\left(E_{D D E A}\right) * 100\right\} /\left(T_{D D E A}+T_{L}\right) \ldots \ldots . .(2) \\
& \operatorname{EE}(\%)=\left\{\left(E_{D D E A}\right) * 100\right\} / T_{D D E A}
\end{aligned}
$$

Where $\mathrm{T}_{\text {DDEA }}=$ Total amount of DDEA present in SLN, $\mathrm{T}_{\mathrm{L}}=$ Total amount of lipid

\section{In vitro release study of DDEA-SLN}

SLN dispersion equivalent to $58.03 \mathrm{mg}$ of DDEA was placed in pretreated dialysis bag dipped in $200 \mathrm{ml}$ of dissolution medium (phosphate buffer $\mathrm{pH}$ 6.8) at 50 $\mathrm{rpm}$ and $37 \pm 0.5^{\circ} \mathrm{C}$. Samples withdrawn were analyzed spectrophotometrically.

\section{Ex vivo permeation study of DDEA-SLN}

buccal mucosa was mounted over the Franz diffusion cell (Electro Lab EDC 07, India), diffusion area: $1.77 \mathrm{~cm}^{2}$, receptor media: $12 \mathrm{ml}$ of phosphate buffer $\mathrm{pH} 7.4$, at 50 $\mathrm{RPM}$ and $37 \pm 1^{\circ} \mathrm{C} .1 \mathrm{~h}$ SLN dispersion equivalent to 58.03 $\mathrm{mg}$ of DDEA was placed in the donor.

\section{Preparation of DDEA SLN loaded TP}

TP was prepared with HPC LF (4\% w/v), (0.1\% w/v), LPG $(15 \% \mathrm{w} / \mathrm{w})$ and DDEA-SLN $(53.93 \% \mathrm{w} / \mathrm{w})$ dispersed in Milli Q water, stirred and casted over the backing layer. Briefly the backing layer was papered with EC (4\% w/v) and DBP $(30 \% \mathrm{w} / \mathrm{w})$ dissolved in EA and acetone $(1: 1,8$ $\mathrm{ml})$. The dried TP was packed in self sealing plastic bags in a dissector.

\section{Evaluation of the TP}

TP was evaluated for appearance, weight and thickness uniformity, drug content, folding endurance and surface $\mathrm{pH}$

\section{Swelling index (SI)}

The swelling studies ${ }^{10}$ of TP was carried out in simulated saliva pH 6.8. SI was calculated using the Equation no 4.

$$
S I=\left(W_{t}-W_{0}\right) * 100 / W_{0}
$$

Where $\mathrm{W}_{\mathrm{t}}$ and $\mathrm{W}_{\mathrm{o}}$ are the weights of the patch at time $\mathrm{t}$ and 0 .

\section{In vitro residence time of TP}

orcine buccal mucosa, $(2.5 * 2.5 \mathrm{~cm})$, was glued to the surface of a glass slide, vertically attached to the USP disintegration apparatus containing $800 \mathrm{ml}$ of simulated saliva $\mathrm{pH} 6.8$ at $37^{\circ} \mathrm{C}$. Hydrated TP $\left(1.5^{*} 2 \mathrm{~cm}\right)$ was placed over the mucosal membrane. The glass slide was allowed to move up and down so that the patch was completely immersed at the lowest point and was out at the highest point. The time for complete detachment of the patch was recorded. ${ }^{10}$

\section{Tensile strength}

Tensile tester (H5KS, Tinius Olsen, UK) equipped with a $50 \mathrm{~kg}$ load cell, pneumatic grip and Horizon software was used to determine tensile stress and strain (elongation at break) of the TP(25 $25 \mathrm{~mm}$ area and $0.28 \mathrm{~mm}$ thickness). The grip separation was set at $10 \mathrm{~mm}$ and the crosshead speed was $50 \mathrm{~mm} / \mathrm{min}$ Young Modulus was calculated by the ratio of tensile stress to strain.

\section{Mucoadhesive strength}

Porcine buccal mucosa was glued to a glass slide was the immovable lower jaw TP $(25 * 25 \mathrm{~mm})$ was placed over the mucosa at one end and the other end was fixed to the movable upper jaw. The force required to detach the TP from the mucosa was recorded.

\section{In vitro drug release from TP}

TP $(1.5 * 2 \mathrm{~cm})$ was glued to a glass slide using a cyanoacrylate adhesive over the backing layer so that the mucoadhesive layer faced the dissolution media $(50 \mathrm{ml}$ of simulated saliva $\mathrm{pH} 6.8$ at $37 \pm 0.5^{\circ} \mathrm{C}$ and $50 \mathrm{rpm}$ ) and placed at the bottom of the Jar of USP dissolution apparatus type II (Electrolab TDT-08L, India). Analyzed spectrophotometerically. ${ }^{10}$

\section{Ex vivo permeation study of TP}

TP $(1.5 * 2 \mathrm{~cm})$ was placed in intimate contact of preequilibrated porcine buccal mucosa mounted Franz diffusion cell, effective surface: $4.9107 \mathrm{~cm}^{2}$, receptor media: $50 \mathrm{ml}$ of phosphate buffer $\mathrm{pH} 7.4$, at $37 \pm 1^{\circ} \mathrm{C}$ and $50 \mathrm{RPM}$. Samples were analyzed spectrophotometrically. ${ }^{10}$

\section{FTIR, SEM, DSC and XRD}

FTIR, SEM (Scanning Electron Microscopy), DSC and XRD of the DDEA, compritol, DDEA-SLN and TP were carried out as per standard procedures.

\section{RESULTS AND DISCUSSION}

\section{Solubility of DDEA in Lipid}

Active ingredient solubility in the lipid is important in 
determining drug loading into SLN. ${ }^{11}$ The characteristic melting point peak of DDEA $\left(149.08^{\circ} \mathrm{C}\right)$ had disappeared in the DSC thermogram of melted mixture (Fig 4A). This indicated that DDEA is soluble in Compritol.

\section{Preparation of DDEA-SLN}

DDEA-SLN was non sticky after lyophilization and was stored at $4^{\circ} \mathrm{C}$ in refrigerator. The PS and EE ranged from $75.31 \pm 3.2 \mathrm{~nm}$ (D11) to $606.65 \pm 2.5 \mathrm{~nm}$ (D15) and 15.81 $\pm 3.2 \%$ (D18) to $57.62 \pm 0.5 \%$ (D16) respectively (Table 1). The actual values of independent variables are shown in (Table 2). SLN batch D6 with PS $178.88 \pm 1.3 \mathrm{~nm}$ and EE $52.14 \pm 1.6 \%$ was very close to the point prediction of the CCD. The PDI and ZP of D6 were determined as $0.241 \pm 0.02$ and $-30.333 \pm 0.7 \mathrm{mV}$ respectively. The anionic charge and the value of $\mathrm{ZP}$ are within the limits of non toxicity. ${ }^{13}$ Drug content was between $81.23 \pm 1.2 \%$ (D1) to $96.25 \pm 1.7 \%$ (D17). Percent DL was in the range of 30.81 $\pm 1.52(\mathrm{D} 17)$ and $3.4 \pm 0.12(\mathrm{D} 20)$.

\section{Experimental design}

The mathematical model describing the relationship between variables (A, B and $C$ ) and response PS $\left(Y_{1}\right)$ and $\mathrm{EE}\left(\mathrm{Y}_{2}\right)$ could be reduced to the equations 5 and 6 respectively.

$\left(Y_{1}\right)=161.73+130.04 A-116.43 B+46.02 C-59.43 A B-$ $1.55 A C-102.54 B C+59.01 A^{2}+70.69 B^{2}+10.87 C^{2}$.

\section{In the above equations, $t$}

The impact of the independent variables and their

Table 1: CCD in various runs and coded values, predicted values and percentage prediction error of response $Y_{1}$ and $Y_{2}$

\begin{tabular}{|c|c|c|c|c|c|c|c|c|c|c|c|c|c|}
\hline \multirow[t]{2}{*}{ Run } & \multirow{2}{*}{$\begin{array}{l}\text { Code } \\
\text { of the } \\
\text { SLN } \\
\text { batches }\end{array}$} & \multirow[t]{2}{*}{ Block } & \multicolumn{3}{|c|}{$\begin{array}{l}\text { Independent } \\
\text { variables }\end{array}$} & \multicolumn{2}{|c|}{ Dependent variables } & \multirow[t]{2}{*}{ PDI† } & \multirow[t]{2}{*}{ DL† } & \multicolumn{2}{|c|}{ Predicted values } & \multicolumn{2}{|c|}{$\begin{array}{l}\text { Percentage } \\
\text { prediction erro }\end{array}$} \\
\hline & & & $\bar{A}$ & $\mathrm{~B}$ & $\mathrm{C}$ & Y1† & Y2† & & & $\mathrm{Y1}$ & & Y2 & \\
\hline 1 & D1 & Day1 & -1 & 1 & -1 & $92.11 \pm 2.2$ & $37.06 \pm 1.5$ & $0.33 \pm 0.017$ & $22.87 \pm 1.23$ & 100.94 & 41.41 & -9.59 & -11.75 \\
\hline 2 & D2 & Day1 & 1 & 1 & -1 & $304.07 \pm 3.6$ & $42.71 \pm 0.8$ & $0.26 \pm 0.021$ & $10.22 \pm 0.85$ & 336.34 & 45.90 & -10.61 & 3.78 \\
\hline 3 & D3 & Day1 & -1 & 1 & 1 & $101.94 \pm 2.1$ & $36.00 \pm 1.3$ & $0.23 \pm 0.023$ & $10.33 \pm 0.47$ & 82.087 & 35.49 & 19.47 & 1.40 \\
\hline 4 & D4 & Day1 & 0 & 0 & 0 & $242.88 \pm 2.4$ & $55.27 \pm 0.4$ & $0.26 \pm 0.045$ & $11.22 \pm 0.33$ & 183.52 & 49.25 & 24.43 & 10.88 \\
\hline 5 & D5 & Day1 & 1 & -1 & 1 & $743.56 \pm 2.8$ & $54.15 \pm 2.1$ & $0.11 \pm 0.027$ & $5.46 \pm 0.26$ & 777.01 & 55.99 & -4.49 & -3.41 \\
\hline 6 & D6 & Day2 & 0 & 0 & 0 & $178.88 \pm 1.3$ & $52.14 \pm 1.6$ & $0.24 \pm 0.022$ & $10.65 \pm 0.71$ & 183.52 & 49.25 & -2.59 & 5.53 \\
\hline 7 & D7 & Day2 & -1 & 1 & -1 & $149.61 \pm 2.2$ & $37.48 \pm 1.4$ & $0.27 \pm 0.031$ & $23.07 \pm 1.42$ & 119.01 & 36.89 & 20.45 & 1.56 \\
\hline 8 & D8 & Day2 & 1 & -1 & -1 & $387.28 \pm 2.1$ & $43.29 \pm 1.2$ & $0.36 \pm 0.053$ & $10.35 \pm 0.53$ & 409.97 & 45.05 & -5.86 & -4.07 \\
\hline 9 & D9 & Day2 & -1 & -1 & 1 & $357.58 \pm 2.8$ & $56.74 \pm 1.3$ & $0.24 \pm 0.035$ & $15.37 \pm 0.84$ & 328.14 & 54.80 & 8.23 & 3.41 \\
\hline 10 & D10 & Day2 & 1 & 1 & 1 & $153.21 \pm 2.4$ & $51.28 \pm 2.2$ & $0.25 \pm 0.024$ & $5.19 \pm 0.21$ & 147.21 & 48.18 & 3.91 & 6.04 \\
\hline 11 & D11 & Day2 & 0 & 0 & 0 & $75.31 \pm 3.2$ & $48.53 \pm 0.6$ & $0.35 \pm 0.035$ & $9.98 \pm 0.37$ & 110.51 & 50.78 & -46.75 & -4.64 \\
\hline 12 & D12 & Day2 & 0 & 0 & 0 & $102.41 \pm 2.3$ & $49.18 \pm 0.5$ & $0.28 \pm 0.051$ & $10.11 \pm 0.42$ & 110.51 & 50.78 & -7.91 & -3.25 \\
\hline 13 & D13 & Day3 & 0 & 1.68 & 0 & $179.54 \pm 2.6$ & $35.00 \pm 1.2$ & $0.14 \pm 0.019$ & $7.41 \pm 0.52$ & 195.27 & 36.19 & -8.76 & -3.41 \\
\hline 14 & D14 & Day3 & 0 & 0 & 0 & $96.72 \pm 2.3$ & $37.45 \pm 0.9$ & $0.48 \pm 0.062$ & $7.89 \pm 0.35$ & 191.15 & 43.95 & -97.63 & -17.37 \\
\hline 15 & D15 & Day3 & 0 & -1.68 & 0 & $606.65 \pm 2.5$ & $52.12 \pm 0.6$ & $0.35 \pm 0.085$ & $10.64 \pm 0.76$ & 586.88 & 49.14 & 3.25 & 5.71 \\
\hline 16 & D16 & Day3 & 0 & 0 & 1.68 & $284.97 \pm 2.1$ & $57.62 \pm 0.5$ & $0.27 \pm 0.074$ & $7.11 \pm 0.25$ & 299.31 & 60.42 & -5.03 & -4.86 \\
\hline 17 & D17 & Day3 & 0 & 0 & -1.68 & $162.88 \pm 2.8$ & $54.41 \pm 0.7$ & $0.16 \pm 0.011$ & $30.81 \pm 1.52$ & 144.51 & 49.82 & 11.28 & 8.42 \\
\hline 18 & D18 & Day3 & -1.68 & 0 & 0 & $95.74 \pm 2.3$ & $15.81 \pm 3.2$ & $0.42 \pm 0.057$ & $18.51 \pm 1.38$ & 139.35 & 15.62 & -45.55 & 1.15 \\
\hline 19 & D19 & Day3 & 0 & 0 & 0 & $273.51 \pm 2.6$ & $45.12 \pm 2.2$ & $0.31 \pm 0.069$ & $9.35 \pm 0.37$ & 191.15 & 43.95 & 30.11 & 2.57 \\
\hline 20 & D20 & Day3 & 1.68 & 0 & 0 & $624.41 \pm 2.9$ & $28.38 \pm 2.5$ & $0.11 \pm 0.072$ & $3.40 \pm 0.12$ & 576.76 & 26.78 & 7.63 & 5.63 \\
\hline
\end{tabular}

† Data represent mean $\pm S D,(n=3)$

Table 2 : Actual and coded values of variables used in the CCD and $R^{2}$ values for $Y_{1}$ and $Y_{2}$

\begin{tabular}{|c|c|c|c|c|c|c|}
\hline \multirow[t]{2}{*}{ Independent variables } & \multirow[t]{2}{*}{ Unit } & \multicolumn{5}{|c|}{ Levels } \\
\hline & & $-\alpha$ & -1 & 0 & +1 & $+\alpha$ \\
\hline Lipid (A) & $\%$ & 0.16 & 0.50 & 1.00 & 1.50 & 1.84 \\
\hline Surfactant (B) & $\%$ & 0.08 & 0.10 & 0.13 & 0.15 & 0.17 \\
\hline Volume of Organic Phase (C) & $\mathrm{ml}$ & 4.89 & 10.00 & 17.50 & 25.00 & 30.11 \\
\hline Dependent variables & $\mathbf{R 2}$ & Adjusted R2 & Predicted R2 & $\begin{array}{l}\text { Adequate } \\
\text { precision }\end{array}$ & SD & $\% \mathrm{CV}$ \\
\hline Y1 & 0.9558 & 0.9062 & 0.7121 & 14.4379 & 62.14 & 23.84 \\
\hline Y2 & 0.9082 & 0.8049 & 0.1664 & 11.9526 & 4.84 & 10.88 \\
\hline
\end{tabular}


Malviya, et al.: Diclofenac SLN transmucosal patch

Table 3: ANOVA results for Particle size (Y1) and Entrapment efficiency (Y2) as the response

\begin{tabular}{|c|c|c|c|c|c|c|c|c|c|c|}
\hline \multirow[t]{2}{*}{ Source } & \multicolumn{2}{|c|}{ Sum of Squares } & \multirow[t]{2}{*}{ df } & \multicolumn{2}{|c|}{ Mean Square } & \multicolumn{2}{|c|}{ F Value } & \multicolumn{3}{|c|}{ p-value, Prob $>$ F } \\
\hline & Y1 & Y2 & & Y1 & Y2 & Y1 & Y2 & Y1 & Y2 & \\
\hline Block & 27902.32 & 194.33 & 2 & 13951.16 & 97.16 & & & & & \\
\hline Model & 668591.56 & 1853.06 & 9 & 74287.95 & 205.89 & 19.24 & 8.79 & 0.0002 & 0.0027 & significant \\
\hline A-Lipid & 230957.50 & 150.19 & 1 & 230957.50 & 150.19 & 59.82 & 6.41 & $<0.0001$ & 0.0351 & \\
\hline B-Surfactant & 185121.25 & 202.30 & 1 & 185121.25 & 202.30 & 47.95 & 8.64 & 0.0001 & 0.0187 & \\
\hline $\begin{array}{l}\text { C-Organic } \\
\text { phase }\end{array}$ & 28928.71 & 135.56 & 1 & 28928.71 & 135.56 & 7.49 & 5.79 & 0.0256 & 0.0427 & \\
\hline$A B$ & 28253.02 & 35.57 & 1 & 28253.02 & 35.57 & 7.32 & 1.51 & 0.0269 & 0.2527 & \\
\hline$A C$ & 19.14 & 0.18 & 1 & 19.14 & 0.18 & 0.00 & 0.01 & 0.9456 & 0.9306 & \\
\hline$B C$ & 84107.41 & 68.73 & 1 & 84107.41 & 68.73 & 21.78 & 2.93 & 0.0016 & 0.1250 & \\
\hline$A^{2}$ & 50144.46 & 931.86 & 1 & 50144.46 & 931.86 & 12.99 & 39.81 & 0.0069 & 0.0002 & \\
\hline$B^{2}$ & 71948.90 & 2.98 & 1 & 71948.90 & 2.98 & 18.63 & 0.12 & 0.0026 & 0.7302 & \\
\hline$C^{2}$ & 1702.82 & 224.46 & 1 & 1702.82 & 224.46 & 0.44 & 9.58 & 0.5253 & 0.0147 & \\
\hline Residual & 30888.59 & 194.33 & 8 & 3861.07 & 23.41 & & & & & \\
\hline Lack of Fit & 12846.64 & 1853.06 & 5 & 2569.33 & 30.55 & 0.42 & 2.65 & 0.8098 & 0.2257 & $\begin{array}{l}\text { not } \\
\text { significant }\end{array}$ \\
\hline Pure Error & 18041.95 & 150.19 & 3 & 6013.98 & 11.50 & & & & & \\
\hline Cor Total & 727382.48 & 202.30 & 19 & & & & & & & \\
\hline
\end{tabular}

interaction on the selected responses is obtained from the magnitude of the coefficients and their mathematical sign.

From the ANOVA results, the model was highly statistically significant with $\mathrm{p}<0.05$. (Table 3) The lack of fit (F-values) was insignificant relative to the pure error and the chance of their occurrence due to noise is minimum. ${ }^{14}$ The significant values were of three linear factors $(\mathrm{A}, \mathrm{B}, \mathrm{C})$, two interaction factors $(A B, B C)$ and two quadratic factors $\left(A^{2}, B^{2}\right)$ whereas the insignificant values were of $A C$ and $C^{2}$ in case of PS. The significant values in case of $\mathrm{EE}$ were of $\mathrm{A}, \mathrm{B}, \mathrm{C}, \mathrm{A}^{2}$ and $C^{2}$ whereas the insignificant values were of $A B, A C, B C$ and $\mathrm{B}^{2}$. The predicated $\mathrm{R}^{2}$ values; and also the similarities between $\mathrm{R}^{2}$ and adjusted $\mathrm{R}^{2}$ values shows the adequacy of the model to predict the response (Table 2). The values of coefficient of variation (CV\%), which is an estimation of the standard deviation (SD); and the signal to noise ratio of greater than 4 indicates the precision and reliability of the model (Table 2).

The response surface from the 3D plots of interaction shows there was decrease in PS with decrease in $\%$ of lipid, increase in $\%$ of surfactant and decrease in volume of organic phase (Figure 1A:a,b,c). And increase in EE with increase in $\%$ of lipid, decrease in $\%$ of surfactant and increase in volume of organic phase (Figure 1B:d,e,f).

The validation of RSM proves its high predictive ability with low magnitude of prediction error (Figure 1B:g,i); and closeness between anticipated values of the residuals and experimental values (Table 1). ${ }^{14}$ (Figure 1B:h,j). The prediction error for PS and EE was found to be varying between $97.63-30.11 \%$ and $17.37-10.88 \%$ respectively. Point predicted optimized composition process was at lipid $1 \%$, surfactant $0.13 \%$ and volume of organic phase at 4.89 $\mathrm{ml}$ (PS $161.73 \mathrm{~nm}$ and EE 54.41\%).

\section{In vitro drug release of DDEA-SLN}

Batch D6 was studied as it met the desirable criteria of PS and EE. ${ }^{15}$ (Figure 2A), which showed $10.35 \pm 2.1 \%$ release in $30 \mathrm{~min}$ followed by sustained release $(98.26 \pm$ $3.4 \%$ ) at $24 \mathrm{~h}$. The initial burst release was due to drug enriched shells of SLN, ${ }^{16}$ and small size (large surfaces) of the nanoparticles ${ }^{17}$ Kinetic mathematical models predicted the best fit mechanism was Korsemeyer-Peppas diffusion type (Table 4). ${ }^{18}$ The slow release suggests that amorphous DDEA is homogeneously dispersed in the crystalline lipid matrix (confirmed by DSC and XRD studies) thus decreasing their diffusional mobility. DDEA dissolves in lipid, diffuses to the surface; undergoing partitioning between lipid and aqueous phase and is dialyzed into the medium..$^{19}$ Also due to large drug loading the degree of diffusion decreases since too many molecules trying to diffuse and limit their own permeation. ${ }^{20}$

\section{Ex vivo permeation studies of DDEA-SLN}

Due to morphological similarities, buccal mucosa of the pig is considered as an appropriate model of human buccal mucosa for drug permeability studies. ${ }^{21}$ Membrane coating granules (MCGs) are the principle penetration barrier which 

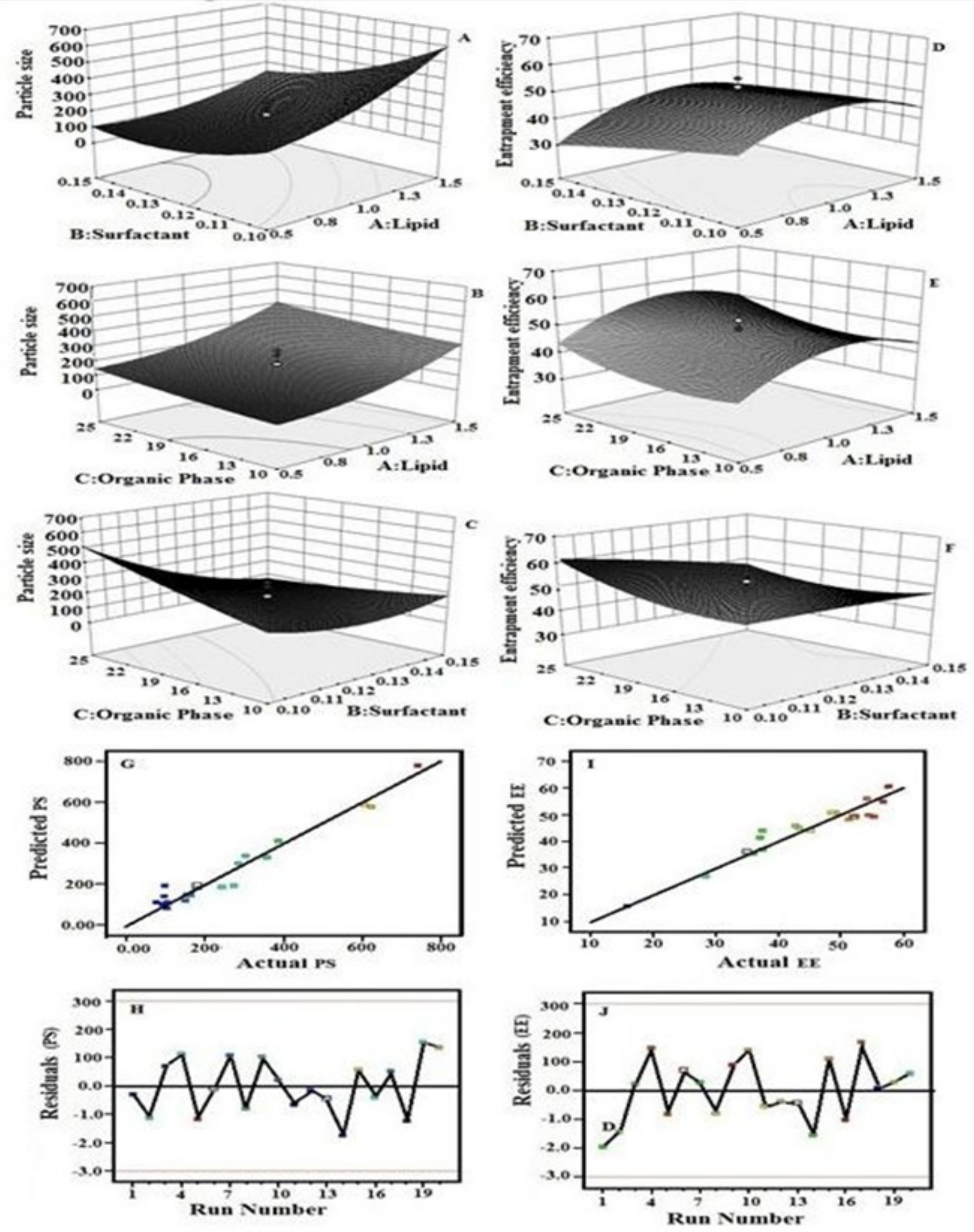

Figure 1A: 3D plots showing the effect of independent variables on Response $\left(Y_{1}\right)$ and $\left(Y_{2}\right) .(1 B):(a)$ and $(b)$ Linear correlation plot between actual and predicted values corresponding residual plot for response $\mathrm{Y}_{1}$. (c) and (d) Linear correlation plot between actual and predicted values corresponding residual plot for response $\mathrm{Y}_{2}$ 

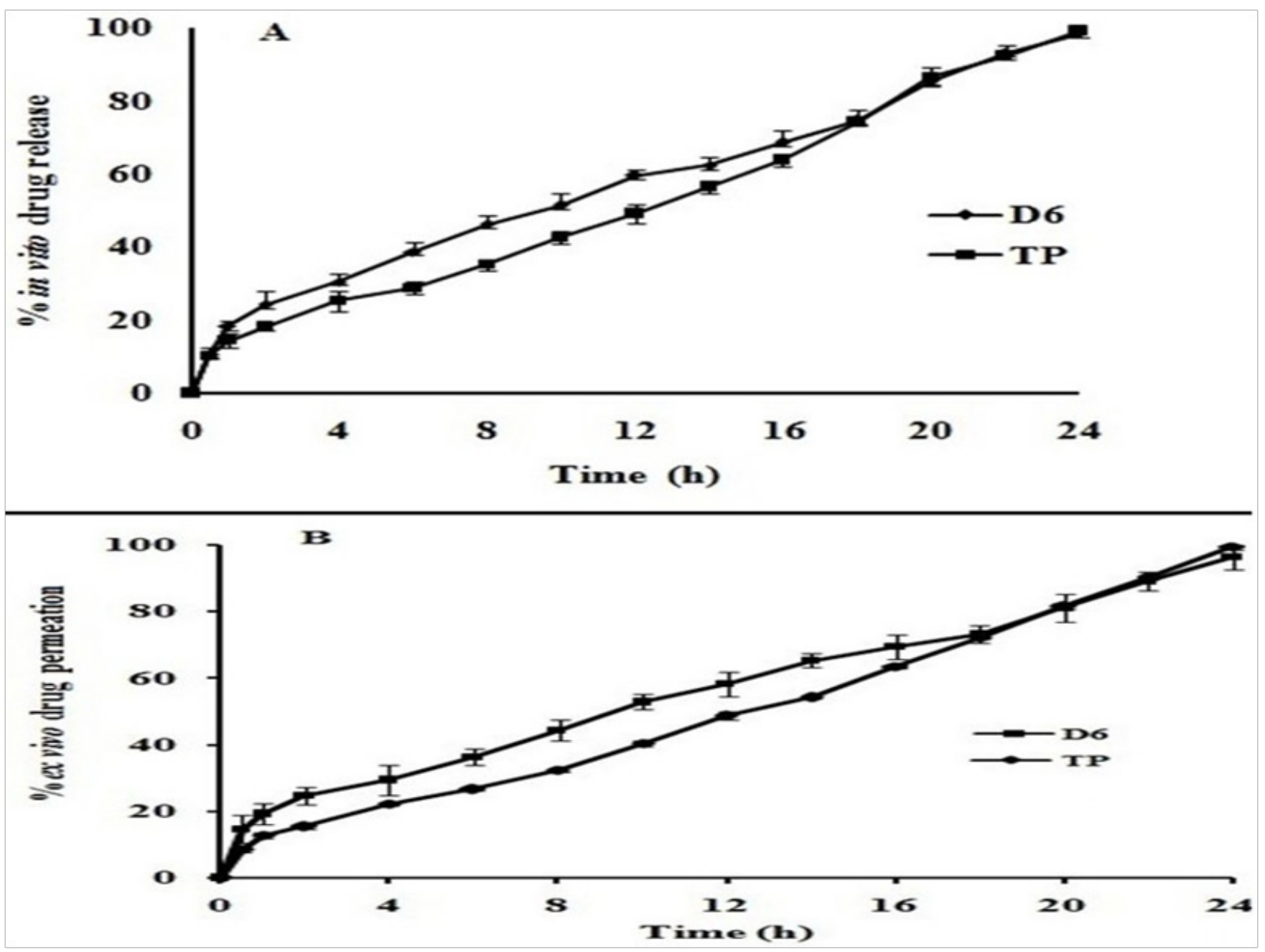

Figure: (2A) In vitro release study D6 and TP in simulated saliva pH 6.8 : (2B) Ex vivo release study of the batch D6 and TP in phosphate buffer pH 7.4

\begin{tabular}{|c|c|c|c|c|c|c|c|c|}
\hline \multirow{2}{*}{$\begin{array}{l}\text { SLN / Patch } \\
\text { code }\end{array}$} & \multirow[t]{2}{*}{ Zero order } & \multirow[t]{2}{*}{ First order } & \multirow[t]{2}{*}{ Matrix } & \multicolumn{2}{|c|}{ Krosmeyer-Peppas } & \multirow{2}{*}{$\begin{array}{l}\text { Hixon- } \\
\text { Crowell }\end{array}$} & \multirow[t]{2}{*}{ Higuchi } & \multirow{2}{*}{$\begin{array}{l}\text { Best fit } \\
\text { model }\end{array}$} \\
\hline & & & & $\mathbf{R}$ & $\mathbf{n}$ & & & \\
\hline D6 & 0.7117 & 0.8834 & 0.8557 & 0.9739 & 0.4635 & 0.8028 & 0.9451 & Peppas \\
\hline TP & 0.9342 & 0.9317 & 0.9768 & 0.9811 & 0.4515 & 0.9712 & 0.8627 & Peppas \\
\hline
\end{tabular}

spread their lipid content into the intercellular space..$^{22}$ and prevent the movement of xenobiotics in the mucosa. The drug permeated is $96.28 \pm 3.5 \%$ at $24 \mathrm{~h}$ (Figure $2 \mathrm{~B}$ ) which could be due to DDEASLN easily bypass the protective barrier of MCGs. Good correlation was obtained between In vitro and ex vivo studies with slow initial release followed by sustained release.

\section{Evaluation of TP}

TP was opaque, elegant and smooth with average weight and thickness of one sq $\mathrm{cm}$ being $0.082 \pm 0.0015 \mathrm{~g}$ and $0.24 \pm 0.024 \mathrm{~mm}$ respectively. Content uniformity was in the range of $97.33 \pm 1.05 \%$. Folding endurance was above
300 which prove the integrity of the patch when subjected to stress conditions. The surface $\mathrm{pH}$ was within the range of salivary $\mathrm{pH}$ (6.5 to 7.4$)$ which assures non irritancy.

Swelling Index (SI) of TP

The SI decreased with incorporation of SLN $5.35 \pm$ $0.47 \%$ at $15 \mathrm{~min}$. Hydrophilic polymers HPC-LF and Polycarbophil swell considerably and an increase in SI was observed with time.

\section{In vitro residence time of TP}

The in vitro residence time for TP was $24.16 \pm 0.17 \mathrm{~h}$. 
HPC-LF is non ionic water soluble cellulose ether with a viscosity ranging $75150 \mathrm{cps}$ at $5 \% \mathrm{w} / \mathrm{v}$ which imparts reasonable mucoadhesion. $\mathrm{PC}$ is a high molecular weight acrylic acid polymer cross linked with divinyl glycol which extracts water from the mucous layer and creates strong hydrogen bonds responsible for the mucoadhesion.

\section{Tensile strength}

The values of tensile strength $8.43 \pm 0.1 \mathrm{Kg} / \mathrm{mm}^{2}$, Young's modulus $24.58 \pm 0.68 \mathrm{Kg} / \mathrm{mm}^{2}$ and percent elongation (34.33 \pm 0.57$)$ display ductile patch with low brittleness, strong enough to prevent rupture during the cutting and packaging processes. This can be attributed to the oxygen group (-O-) present in the HPC-LF and PC polymer backbone and also in the plasticizer LPG, which tends to reduce chain stiffening thus imparting mechanical properties of softness and durability.

\section{Mucoadhesive strength}

Mucoadhesion occurs with intimate contact of polymer and mucosa as a result of good wetting of the surface with saliva. The intensity of the adhesion is mainly affected by the swelling capacity of the patch. The prepared TP was appropriate with a high mucoadhesive strength $(0.039 \mathrm{Kg})$ and hence low possibility of easy removal.

\section{In vitro release of TP}

A higher rate of drug released (10\%) in short time of 30 min representing immediate release with no lag time (Figure 2A). LPG as plasticizer could reduce the glass transition temperature, making the gel rubbery and disorganizing the polymer chain network thus improving the release rate. The drug release was prolonged $(99.22 \pm 0.7 \%$ in 24

h) due to dual resistance from SLN and the patch. The

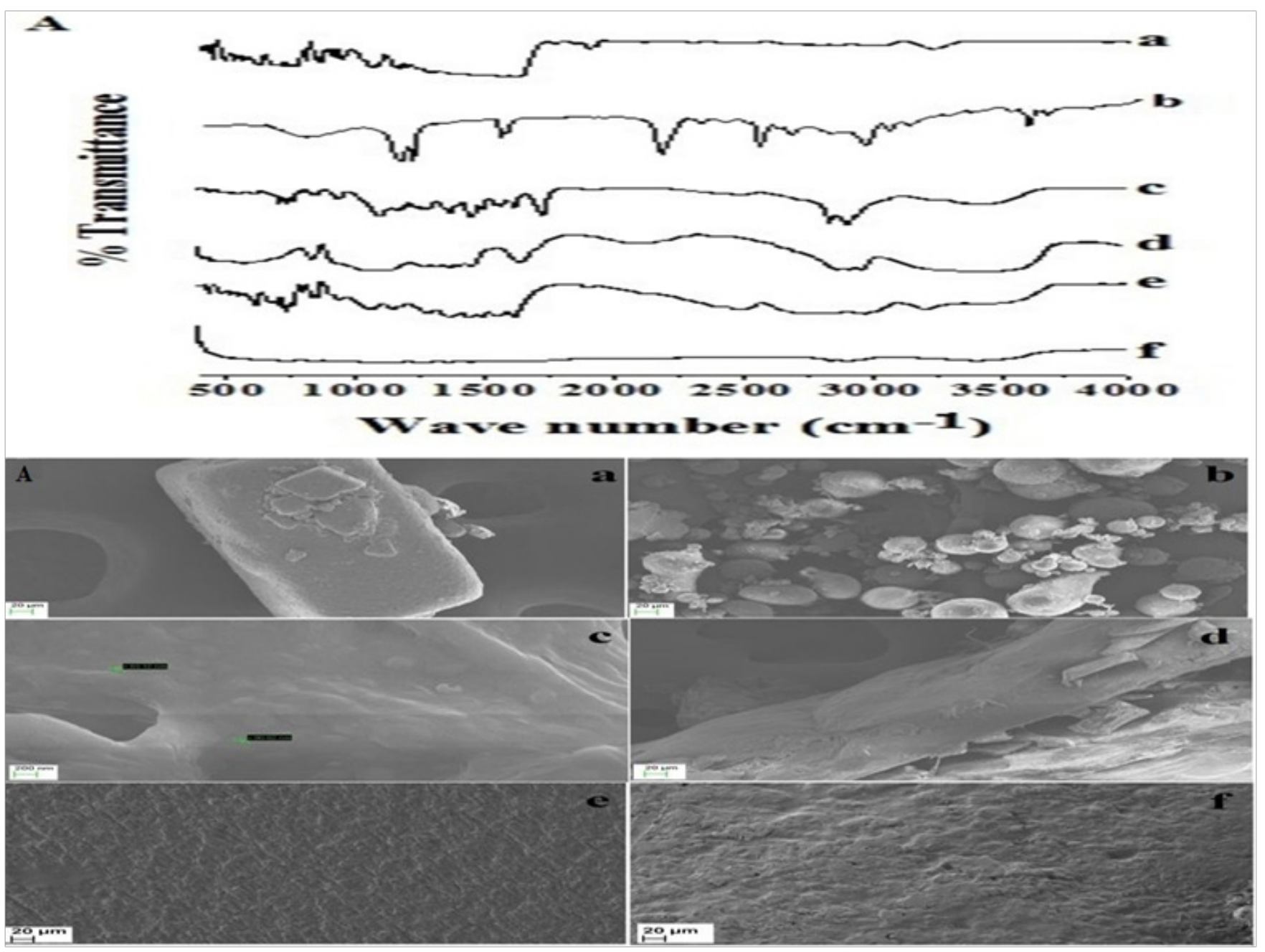

Figure : (3A) FTIR spectrum of a) DDEA b) Compritol ATO 888 c) SLN D6 d) HPC-LF e) Physical mixture of DDEA and HPC-LF f) TP (3B) SEM images of a) DDEA b) Compritol ATO 888 c) SLN D6 d) HPC-LF e) Dummy patch of HPC-LF f) TP 
mechanism of release was found to be the KorsemeyerPeppas diffusion type (Table 4) as determined by PCP disso software. Analgesic effect corresponds to onset of action with minimum effective concentration (MEC) of Diclofenac $(50 \mathrm{ng} / \mathrm{ml})$ which was released in first five min and was maintained for $24 \mathrm{~h}$. With this release profile a decrease in frequency of administration to once a day patch can be achieved.

\section{Ex vivo permeation study of TP}

The effective pore radii of the oral mucosal membrane with respect to the aqueous path is $18-53 \mathrm{~A}^{\circ} \cdot{ }^{23} \mathrm{Buccal}$ mucosa has a negative charge at physiological $\mathrm{pH}$ and dissociation constant of DDEA is 10.75 . This would result in the ionization of drug leading to absorption through the aqueous paracellular pathway and can be correlated to the $10 \%$ immediate release of DDEA in $30 \mathrm{~min}$ (Figure
$2 \mathrm{~B})$. The high permeation $(99.53 \pm 0.9 \%)$ in $24 \mathrm{~h}$ could be attributed to the PS $(178 \mathrm{~nm})$ of the designed SLN passively diffusing through transcellular pathway. Also fabrication of the lipoidal DDEA in to SLN resulted in the carrier sustaining the drug release for a period of 24 h. Incidentally the amount of drug permeated in first five minutes was well above the MEC of Diclofenac, which was also maintained throughout $24 \mathrm{~h}$.

\section{FTIR, SEM, DSC and XRD}

The characteristic IR $(\mathrm{KBr})$ peaks of DDEA appearing at $3222.83 \mathrm{~cm}^{-1}$ (NH stretching of secondary amine), $1563.95 \mathrm{~cm}^{-1}$ (-C=O stretching of the carboxyl ion) and $744.47 \mathrm{~cm}^{-1}$ (C-Cl stretching) were intact in the IR spectra of DDEA-SLN and the TP inferring that the drug is compatible with excipients (Figure 3A). The SEM images (Figure 3B) show irregular shape of DDEA and compritol

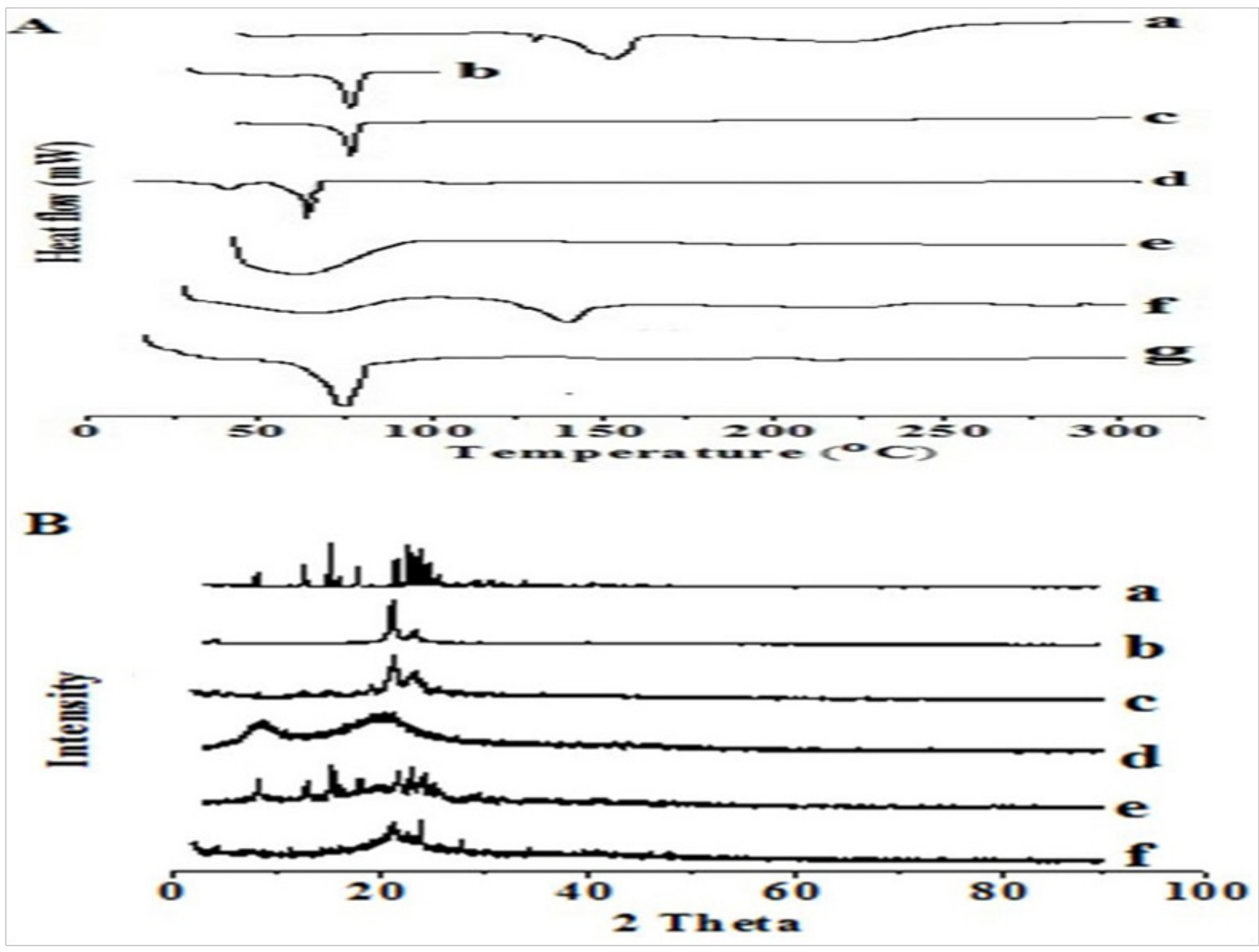

Figure :(4A) DSC thermograms of a) DDEA b) Compritol ATO 888 c) melted mixture of Compritol ATO 888 and DDEA d) SLND6e) HPC -LF f) Physical mix of DDEA and HPC g) TP (4B) XRD thermograms of a) DDEA b) Compritol ATO 888 c) SLN D6 d) HPC-LF e) Physical mixture of DDEA and HPC-LF f) TP 
whereas DDEA-SLN have smooth and porous surface existing as spherical (126-220 nm). DDEA-SLN in the TP is evenly distributed when compared to the dummy patch. DSC thermograms (Figure 4A) with endothermic characteristics peak of anhydrous DDEA at $149.08^{\circ} \mathrm{C}$, compritol $\left(70^{\circ} \mathrm{C}\right)$ and DDEA-SLN $\left(65^{\circ} \mathrm{C}\right)$ indicate crystallinity. Shift of the peaks of DDEA and compritol to a lower temperature suggest an interaction leading to SLN formation with nanocrystalline lipids in the SLN. Sharp peak of SLN indicates that DDEA located in the core of the SLN exists in amorphous state within the crystalline lipid matrix. All these confirm that crystalline substance requires more energy for the melting to overcome lattice force. ${ }^{24}$ The broad melting point peak of the TP indicates that the HPC -LF exists as amorphous form in the patch. Diffraction patterns of DDEA, compritol, DDEA-SLN and TP (Figure 4B) confirms the results of DSC studies. Compritol has sharp peaks that which is also present in the diffractograms of the SLN indicating the ordered crystalline arrangements. ${ }^{25}$ Disappearance of sharp DDEA peaks in the SLN and in the patch indicates that it exists as amorphous form.

\section{CONCLUSION}

DDEA-SLN was successfully prepared by SEET and optimized using CCD. Batch D6 had the optimum value as desired and as point predicted for PS and EE. The developed DDEA-SLN is promising for the local delivery at the gingival tissue as the drug release is controlled by the delivery system. Further the DDEA-SLN was incorporated into TP prepared with film forming and mucoadhesive polymers HPC-LF and polycarbophil respectively. Ex vivo permeation studies of the patch revealed distinct pattern with initial 10\% permeation within 30 min followed by sustained release and permeation for $24 \mathrm{~h}$ attributed to the association of DDEA with a lipophilic carrier Compritol and the second control that is the patch itself, which is also expected to increase the resistance of the system. Thus, effective pain management is possible by application of the developed DDEA-SLN loaded TP at the gingival mucosa of the affected tooth which has the advantage of by passing first pass metabolism and overcoming gastric irritation, resulting in local analgesic action for the sustained period of time with early onset for the treatment of acute and chronic pain. The therapy of once a day application, would also overcome the drawback of increased frequency of administration of DDEA. The prepared patches display required mucoadhesion, which is important for prolonging the residence of the TP on the mucosa. Unidirectional release from the developed patch was ensured with the presence of a backing layer of EC. Therefore the developed system appears very promising for post operative dental pain management.

\section{ACKNOWLEDGMENTS}

Research grants received from DST India, IPA Mumbai and RGUHS Bangalore. The authors are thankful to Prof. BG Shivanada, Then Principal, AACP, Bangalore., Dr. Girish Kunte and Dr Sandhya IISc, Bangalore.

\section{CONFLICT OF INTEREST}

The authors report no conflicts of interest.

\section{REFERENCES}

1. Meechan GJ. Effective topical anesthetic agents and techniques. Dent Clin North Am. 2002;46:759-766.

2. Elliot KH, Paul AM, Gilbert LR. Over-the-counter Analgesics and Antipyretics: $A$ Critical Assessment. Clin Ther. 2000;22:500-548.

3. Ho LW, Reina B, Andrew MR, Yongqiang L, Xiao YW. Chemotherapy with anticancer drugs encapsulated in solid lipid nanoparticles. Adv Drug Deliv Rev. 2007;59:491-504.

4. Subedi RK, Kanga KW, Choi HK. Preparation and characterization of solid lipid nanoparticles loaded with doxorubicin. Eur J Pharm Sci. 2009;37:508-513.

5. Yan F, Chao Z, Zheng Y, Lin M, Lina T, Cunxian S. The effect of poloxamer 188 on nanoparticle morphology, size, cancer cell uptake, and cytotoxicity. Nanomedicine: Nanotechnology Biology and Medicine. 2010;6:170-178

6. Molpeceres J, Guzman M, Aberturas MR, Chacon M, Berges L. Application of central composite designs to the preparation of polycaprolactone nanoparticles by solvent displacement. J Pharm Sci. 1996;85:206-213.

7. Rahman Z, Zidan AS, Khan MA. Non-destructive methods of characterization of Risperidone solid lipid nanoparticles. Eur J Pharm Biopharm. 2010;76:127-137.

8. Kesavan B, Jayaraman A, Velayutham R, Vobalaboina V, Yamsani MR. Lipid nanoparticles for transdermal delivery of flurbiprofen: formulation, in vitro, ex vivo and in vivo studies. Lipids Health Dis. 2009;8:1-15

9. Muller RH, Maeder K, Gohla S. Solid lipid nanoparticles for controlled drug delivery-a review of the state of the art. Eur J Pharm Biopharm. 2000;50:161-177.

10. Supriya SS, Nilesh SS, Sagar S, Vilasrao K. Mucoadhesive Bilayered Patches for Administration of Sumatriptan Succinate. AAPS PharmSciTech. 2008;9:909-916.

11. Mehnert W, Mader K. Solid lipid nanoparticles: Production, characterization and applications. Adv Drug Deliv Rev. 2001;47:165-196.

12. Kuo YC, Chung JF. Physicochemical properties of nevirapine-loaded solid lipid nanoparticles and nanostructured lipid carriers. Colloids Surf B Biointerfaces. 2011;83:299-306.

13. Chrysantha F, Rainer HM. Effect of light and temperature on zeta potential and physical stability in solid lipid nanoparticle $\left(\mathrm{SLN}^{\mathrm{TM}}\right)$ dispersions. Int J Pharm. 1998;168:221-229

14. Joana FF, Tatiana A, Maria A, Maria LG, Selma B, Eliana SB. Experimental factorial design applied to mucoadhesive lipid nanoparticles via multiple emulsion process. Colloids Surf B: Biointerfaces. 2012;100:84-89.

15. Silva AC, Amarala MH, Gonzalez-Mirac E, Santosa D, Ferreiraa D. (2012). Solid lipid nanoparticles-based hydrogels as potential carriers for oral transmucosal delivery of Risperidone: Preparation and characterization studies. Colloids and Surf B: Biointerfaces 2012;93:241-248.

16. Souto EB, Wissing SA, Barbosa $\mathrm{CM}$, Müller RH. Development of a controlled release formulation based on SLN and NLC for topical clotrimazole delivery. Int J Pharm. 2004;278:71-77.

17. Kheradmandnia S, Ebrahim Vasheghani FE, Mohsen N, Fatemeh A. Preparation and characterization of ketoprofen-loaded solid lipid nanoparticles made from beeswax and carnauba wax. Nanomedicine: Nanotechnology Biology and Medicine. 2010;6:753-759.

18. Su L, Zhaoshuai J, MeiJuan Z, Nie X, Yijie S, Gang C. Preparation, Characterization, Pharmacokinetics and Tissue Distribution of Solid Lipid Nanoparticles Loaded with etrandrine. AAPS PharmSciTech. 2011;12:1012-1018.

19. Venkateswarlu $V$, Manjunath $K$. Preparation, characterization and in vitro release 
kinetics of clozapine solid lipid nanoparticles. J Control Release. 2004;95:627-638.

20. Wissing SA, Muller RH. Solid lipid nanoparticles as carrier for sunscreens: in vitro release and in vivo skin penetration. J Control Release. 2002;81:225-233

21. Ra S, Yunmei S, Peddie F, Evans AE. Particle size reduction to the nanometer range: a promising approach to improve buccal absorption of poorly water-soluble drugs. Int J Nanomedicine. 2011;6:1245-1251

22. Wertz PW, Squier CA. Cellular and molecular basis of barrier function in oral epithelium. Crit Rev Ther Drug Carrier Syst. 1991;8:237-269.
23. Tarun Goswami, Bhaskara R Jasti, Xiaoling Li. Estimation of the theoretical pore sizes of the porcine oral mucosa for permeation of hydrophilic permeants. Arch Oral Biol. 2009;54:577-82.

24. Mangesh RB, Varsha $P$, Ashwini $M$, Nilam $P$ Nilkanth $P$. Preparation and Evaluation of Miconazole Nitrate-Loaded Solid Lipid Nanoparticles for Topical Delivery. AAPS PharmSciTech. 2009;10:289-296.

25. Abdelbary G, Rania HF. Diazepam-Loaded Solid Lipid Nanoparticles: Design and Characterization. AAPS PharmSciTech. 2009;10:211-219. 